Introduction Recurrent vulvovaginal candidiasis (RVVC) results in significant physical, financial and psychological sequelae for women, and many women report that VVC affects their intimate relationships. The aetiology of RVVC remains uncertain, and some studies suggest sexual intercourse may be responsible for transmission of Candida species. No publications have documented the affect of sexual intercourse on vaginal candida colonisation.

Methods Fifty nine participants who were culture positive for Candida spp. at screening took part in a randomised controlled trial investigating the effect of oral garlic and placebo on vaginal candidal colonisation. Participants self-collected daily vaginal swabs during the two weeks before menstruation. They kept a daily diary and recorded incidence of sexual intercourse and abnormal vaginal symptoms. Swabs were analysed for quantitative colony counts of candida before and after sexual intercourse.

Results There were 149 episodes of sexual intercourse in participants reporting sexual activity $(n=38)$ over the two week study period. Colonisation levels rose the day following sexual intercourse in 51 episodes, and fell in 56 episodes. In 42 episodes of sexual intercourse, the levels remained the same or women were culture negative on the day following and two days following sexual intercourse. On fifty occasions women had symptoms (itch, abnormal vaginal discharge) on the day of sexual intercourse, and 41 women reported abnormal symptoms two days after sexual intercourse. In 75 episodes, there were no abnormal symptoms the day of, or the day following sexual intercourse.

Conclusion In this study, sexual intercourse, colonisation levels and abnormal vaginal symptoms appeared to be unrelated. Further investigation is recommended into dyspareunia and abnormal vaginal symptoms following sexual intercourse experienced by women with RVVC.

Disclosure of interest statement No pharmaceutical grants were received in the development of this study.

\section{P06.15 THE STABILITY OF THE VAGINAL MICROBIOME IN RELATION TO NEW SEXUAL EXPERIENCES}

1,2) Twin, ${ }^{3,4,5} \mathrm{CS}$ Bradshaw, ${ }^{3}$ LA Vodstrcil, ${ }^{1,2,4} \mathrm{SM}$ Garland, ${ }^{3,5} \mathrm{CK}$ Fairley, ${ }^{3} \mathrm{G}$ Fehler, ${ }^{4} \mathrm{JA}$ Hocking, ${ }^{5} \mathrm{M}$ Law, ${ }^{3} \mathrm{KA}$ Fethers, ${ }^{1,2,4} \mathrm{SN}$ Tabrizii*. ${ }^{1}$ Royal Women's Hospital; ${ }^{2}$ Murdoch Children's Research Institute; ${ }^{3}$ Melbourne Sexual Health Centre; ${ }^{4}$ University of Melbourne; ${ }^{5}$ Monash University, Kirby Institute

\subsection{6/sextrans-2015-052270.316}

Introduction The vaginal microbiome in healthy asymptomatic women can vary greatly, though in most is typically dominated by lactic acid producing Lactobacillus spp. which convey protection against pathogens. Few studies have examined genital bacterial communities in sexually-inexperienced women over time and particularly changes occurring upon initiation of new sexual experiences.

Methods Overall192 samples from 45 healthy women were selected as a subset of a study investigating the sexual health of 17-21 year old university students. Women who were selected included those with no previous sexual experience $(\mathrm{n}=17)$, women experienced in non-coital sexual activities only $(\mathrm{n}=15)$ and women who had engaged in penile-vaginal sex $(n=13)$. The selected participants provided self-collected vaginal swab samples every 3 months for 12 months. Bacterial communities were analysed using Roche 454 amplicon sequencing with PCR primers targeting the V3/4 variable region of the $16 \mathrm{~S}$ rRNA gene.
Results Overall, healthy young women had differing vaginal community states. Onset of non-coital and coital sexual activities does not exert a significant effect on the composition of vaginal bacterial communities. The vaginal community-state with the greatest stability over time consisted of states predominated by L. crispatus and L. iners with median Bray-curtis dissimilarity values of 12.4 and 17.6 respectively ( $\mathrm{p}=0.005$ and 0.024 ). Vaginal microbiomes dominated by other Lactobacillus spp. and non-Lactobacillus spp. gave rise to the most variability over time (dissimilarity values of 41.1 and 66.8). Non-coital and coital sexual activity within this subset of participants did not have any significant effect upon the stability of vaginal bacterial communities $(p=0.3714)$.

Conclusion L. crispatus and L. iners are most commonly found to dominate sexually inexperienced women and convey the most stable environment over time. The initiation of new sexual activities does not appear to have any persistent effect on the vaginal microbiome of young women.

Disclosure of interest statement None to declare.

\section{P07 - STI/HIV diagnosis}

\section{P07.01 MYCOPLASMA GENITALIUM TESTING PATTERN AND INFECTION RATES OVER A SIX-YEAR PERIOD IN MELBOURNE, AUSTRALIA}

${ }^{1,2,3} \mathrm{SN}$ Tabrizi* ${ }^{2}{ }^{2} \mathrm{AM}$ Costa, ${ }^{2} \mathrm{~A}$ Marceglia, ${ }^{1,2} \mathrm{DA}$ Machalek, ${ }^{4} \mathrm{CS}$ Bradshaw, ${ }^{4} \mathrm{M}$ Chen, ${ }^{4} \mathrm{CK}$ Fairley, ${ }^{1,2,3} \mathrm{SM}$ Garland. 'Murdoch Childrens Research Institute; ${ }^{2}$ The Royal Children's and the Royal Women's Hospitals; ${ }^{3}$ University of Melbourne; ${ }^{4}$ Melbourne Sexual Health Centre

\subsection{6/sextrans-2015-052270.317}

Introduction Mycoplasma genitalium ( $\mathrm{Mg})$ is an emerging sexually transmitted pathogen with a strong association with urethritis, cervicitis and pelvic inflammatory disease. Detection of this bacterium using molecular assays has been limited due to lack of readily available commercial assays. However, in house $16 \mathrm{~S}$ rRNA gene qPCR assays have been in use at the laboratory located at the Royal Women's Hospital, in Melbourne Australia for detection of $\mathrm{Mg}$ since 2009. The aim of this study was to analyse $\mathrm{Mg}$ testing patterns and infection rates over this 6 year period.

Methods We analysed overall detection rates and site-specific positivity in clinical specimens received for testing for $\mathrm{Mg}$ between 1 January 2009 and 31 December 2014 from clinics at the Royal Women's Hospital and Melbourne Sexual Health Centre.

Results A total of 46,112 specimens were tested for $\mathrm{Mg}$; 2,853 $(6.2 \%)$ samples were tested in 2009 with an increasing trend to $13,133(28.5 \%)$ in 2014 ( $\mathrm{p}$-trend <0.001). In total $54.7 \%$ were urine samples, $37.7 \%$ vaginal/cervical swabs and $7.6 \%$ were anal, urethral or from other non-specified sites. Overall positivity across all samples was 4.5\% (95\% CI: 4.3-4.7) without any significant change per annum ( $\mathrm{p}$-trend $=0.206$ ). Overall, $\mathrm{Mg}$ detection rate was highest in urethral $(9.0 \%, 95 \%$ CI: $6.7-11.7)$ and anal swabs $(8.8 \%, 95 \%$ CI $6.8-11.1)$ followed by urine (5.8\%, 95\% CI: 5.5-6.7) and cervical/vaginal samples (2.6\%, 95\% CI: $2.4-2.8$ ) ( $\mathrm{p}<0.001)$. A significant increase in positivity was observed in anal swabs, from $2.5 \%$ in 2009 to $12.7 \%$ in 2014 (p-trend $=0.005)$.

Conclusion Increased testing for $\mathrm{Mg}$ by $\mathrm{qPCR}$ has resulted in detection and treatment of over 2000 infections since 2009 in 